Documentation et bibliothèques

DOCUMENTATION BIBLIOTHËQUES

\title{
Georges-Émile Marquis (1878-1960) : un bibliothécaire dynamique mais rébarbatif
}

\section{Gaston Bernier}

Volume 58, numéro 2, avril-juin 2012

URI : https://id.erudit.org/iderudit/1028910ar

DOI : https://doi.org/10.7202/1028910ar

Aller au sommaire du numéro

\section{Éditeur(s)}

Association pour l'avancement des sciences et des techniques de la documentation (ASTED)

\section{ISSN}

0315-2340 (imprimé)

2291-8949 (numérique)

Découvrir la revue

\section{Citer ce document}

Bernier, G. (2012). Georges-Émile Marquis (1878-1960) : un bibliothécaire dynamique mais rébarbatif. Documentation et bibliothèques, 58(2), 77-83. https://doi.org/10.7202/1028910ar

Tous droits réservés (C) Association pour l'avancement des sciences et des techniques de la documentation (ASTED), 2012
Ce document est protégé par la loi sur le droit d'auteur. L'utilisation des services d'Érudit (y compris la reproduction) est assujettie à sa politique d'utilisation que vous pouvez consulter en ligne.

https://apropos.erudit.org/fr/usagers/politique-dutilisation/ 


\section{Georges-Émile Marquis (1878-1960) : un bibliothécaire dynamique mais rébarbatif}

\author{
GASTON BERNIER
}

GastonBernier@assnat.qc.ca

\section{L}

E PARlement QUÉBÉcoIs a connu depuis 1792 une suite " généalogique » de quinze conservateurs ou directeurs de la bibliothèque, de Jacques Langlois (1829-1832) à Hélène Galarneau (2010-). Les personnalités sont fort différentes les unes des autres, comme la formation et les réalisations de chacun. Les uns ont été des essayistes remarqués, d'autres des écrivains ou de brillants professeurs. Chacun a pris sa place dans un moment de l'histoire documentaire.

Le dixième conservateur de la bibliothèque des parlementaires fut Georges-Émile Marquis (1878-1960). S'il a échoué à réaliser bien des projets, il a entrevu des échappées que ses descendants ont fait leurs et que le temps a concrétisé. On trouvera ici une courte biographie du bibliothécaire, un aperçu de son apport, de ses échecs et des éclats qui ont marqué sa carrière.

Georges-Émile Marquis est né le 15 août 1878 à Saint-Pierre-de-la-Rivière-du-Sud ${ }^{1}$ (Montmagny). Ses parents étaient François Marquis et Clorinde Tanguay. Il fit ses études à l'école modèle ${ }^{2}$ de Saint-Gervais (Bellechasse) et à l'École normale Laval à partir de 1896 , et enseigna ensuite pendant cinq ans, d'abord à Saint-Roch-des-Aulnaies puis à Québec. En 1905, il fut nommé inspecteur d'école dans la vallée de la Matapédia. Neuf ans plus tard, en 1914, il devint directeur du Bureau des statistiques (BSQ), mis sur pied en 1912, où il demeura jusqu'en 1934. Le 19 septembre $1934^{3}$, il fut nommé conservateur de la bibliothèque de la Législature $^{4}$. En 1952, après cinquante ans de travail ${ }^{5}$, il prit sa retraite mais resta actif; il publia Le Régiment de Lévis,

1. On disait à l'époque Saint-Pierre-du-Sud.

2. Information donnée dans la réponse à un questionnaire destiné au Bottin des lettres canadiennes-françaises et adressée à Albert Lévesque, 30 septembre 1935 (Assemblée nationale, Archives Marquis, dossier 1.8).

3. Par décret 2234 daté du 19 septembre. Il prit possession de son bureau le $\mathrm{1}^{\mathrm{er}}$ octobre 1934 (Rapport annuel daté du $1^{\mathrm{er}}$ octobre 1935, p. 1). Le 27 septembre, il avait écrit au premier ministre qu'il était prêt à commencer son travail le $1^{\mathrm{er}}$ octobre, "aussitôt que M. Desjardins sera capable de me donner ses clefs " (Archives Marquis, dossier 1.1). Joseph Desjardins, son prédécesseur, fut malade à la fin de son mandat (voir note 25)

4. Il en fut le dixième depuis 1829. Les deux premiers de la lignée furent Jacques Langlois et Étienne Parent (Deuxième centenaire de la Bibliothèque de l'Assemblée nationale, 1802-2002, Rapport; Québec, la Bibliothèque, 2003, p. 61). On trouvera un aperçu biographique de la lignée sous la plume de Gilles Gallichan dans Bulletin de la Bibliothèque de l'Assemblée nationale, vol. 10, $\mathrm{n}^{\circ}$ 1, avril 1991, p. 3-6 et dans la livraison subséquente. Il eut douze présidents ou orateurs comme supérieur immédiat : cinq du Conseil législatif (de Hector Laferté à Jean-Louis Baribeau) et sept de l'Assemblée (de T.D. Bouchard à Alexandre Taché), sept libéraux et cinq unionistes.

5. "[...] cinq comme instituteur, dix inspecteur d'écoles, vingt statisticien et quinze bibliothécaire [...] Total: cinquante ans" (Rapport annuel daté du 27 février 1951, p. 4). en 1952, Images $d u$ Mexique, en 1955, Les Monuments commémoratifs de Québec, en 1958, et il signa la préface du volume L'École normale Laval; un siècle, de Réal Bertrand, en 1957. Il est mort à Québec le 14 décembre 1960 (Pelletier 1996, 13). Il avait épousé Eugénie Plante en 1905 et le couple eut deux enfants ${ }^{6}$, Gaston et Yvette. La famille vécut au 90 rue Lockwell.

Marquis avait de nombreux champs d'intérêt et d'activité. Il mit sur pied l'École des guides touristiques en 1923 et il en fut le secrétaire par la suite ${ }^{7}$. Il réussit à convaincre le gouvernement d'adopter une loi relative au tourisme (projet de loi $n^{\circ} 207$ de 1937) et, surtout, à y faire insérer une section portant sur les «écoles de tourisme $»^{8}$. Son intérêt pour le tourisme le conduisit à écrire une dizaine de volumes ou plaquettes et à donner des conférences ${ }^{9}$. Il voyageait lui-même beaucoup ${ }^{10}$ et ses voyages ont parfois donné lieu à des publications ${ }^{11}$.

Le bibliothécaire Marquis fit partie de la milice pendant trente ans et il était colonel au moment où il en démissionna, en 1930. Il avait été réorganisateur ${ }^{12}$ et commandant du régiment de Lévis (1920-1925) et commandant de la $13^{\mathrm{e}}$ brigade d'infanterie $\mathrm{e}^{13}$ (19271930) ${ }^{14}$. Marquis était très fier de son titre de colonel ${ }^{15}$. Il corrigeait ses correspondants qui se contentaient de l'appeler lieutenant-colonel ${ }^{16}$.

6. Lettre à Félix Desrochers, 21 février 1938 (Archives Marquis, dossier 1.13). Également, réponse à un questionnaire du Guide du Canada français datée du 25 mai 1946 (Archives Marquis, dossier 3.11).

7. Lettre adressée au ministre Joseph Bilodeau datée du $1^{\text {er }}$ juin 1937 (Archives Marquis, dossier 1.12).

8. Lettre adressée au ministre Joseph Bilodeau datée du $1^{\text {er }}$ juin 1937 (Archives Marquis, dossier 1.12).

9. Les Fortifications de Québec (1923); Québec, son site, ses reliques... (1934); Québec hier et aujourd'hui (pour les touristes; 1939); Les Monuments commémoratifs de Québec (1958).

10. En 1949, «long voyage en Europe " (Archives Marquis, dossier 5.2). Voir aussi Trois générations d'éducateurs (Québec : s.n., 1950) : 60-61.

11. La Culture française dans l'Île Maurice (1952); Images du Mexique (1955).

12. Le Fait français au Canada (Montréal : Société nouvelle de publicité, 1953), p. 315.

13. Les Biographies françaises d'Amérique (Montréal : Les Journalistes associés, 1942), p. 105.

14. Le Fait français..., p. 315.

15. Dans Nos bibliothèques publiques (1925), il a inséré une photo de lui en habi militaire. Sous celle insérée dans La Bibliothèque (1934), on lit «Colonel G.E. Marquis, V.D. ", c'est-à-dire "veteran decoration ». Il se fait désigner «Marquis, G.E., colonel » dans l'annuaire téléphonique de 1951-52.

16. "[...] je ne suis pas lieutenant-colonel, mais colonel [...] ce qui équivaut au titre de curé comparé à celui de vicaire " (Lettre datée du 29 mars 1946 adressée à Georges Maheux du ministère de l'Agriculture; Dossier 3.10). Au greffier du Conseil législatif, R.A. Benoît, il écrit le 3 décembre 1945: "[...] vous me bombardez lieutenant-colonel, ce qui me dégrade, puisque depuis quinze ans, j'ai droit au titre de colonel. » (Dossier 3.10). Jean-Guy Pelletier relève une autre remarque semblable adressée au directeur des archives du Québec (Jean-Guy Pelletier 1996, 12). 


\section{Il a ré-ouvert la porte aux abonnés du public en 1936 (la bibliothèque leur était fermée depuis cinq ans) et il était très fier du nombre de lecteurs extérieurs et de leurs emprunts.}

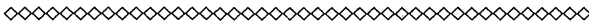

De 1914 à 1934, invité par Lomer Gouin à succéder au fondateur du Bureau des statistiques ${ }^{17}$, Marquis dirigea l'organisme mis sur pied en 1912. Il y rédigea notamment les textes d'introduction de l'Annuaire $d u$ Québec. Il publia d'autres textes sur l'industrie laitière, sur les statistiques ${ }^{18}$, sur l'expansion industrielle ${ }^{19}$, et on garde trace d'une conférence sur l'organisation de la statistique agricole ${ }^{20}$. En 1934, Jean-Charles Harvey, pressenti pour succéder à Joseph Desjardins ${ }^{21}$ à la direction de la bibliothèque mais doublé par Marquis, notera « l'esprit d'organisation et de travail » de son prédécesseur ${ }^{22}$ au BSQ. L'image de fonctionnaire efficace que Marquis y avait laissée donna peut-être au premier ministre Duplessis l'idée de lui offrir la possibilité d'y retourner ${ }^{23}$.

Marquis fut fort actif dans d'autres domaines; un bio-bibliographe a recensé 222 causeries radiophoniques $^{24}$ et environ 375 articles en plus des livres ${ }^{25}$ dont il est l'auteur. Marquis fit partie du bureau de la revue le Terroir et, en 1933, on l'identifiait comme « gérant de la rédaction ${ }^{26}$. Il s'est intéressé à l'histoire ${ }^{27}$ ainsi qu'au

17. G.É. Marquis 1940. Rendez à César (Québec: s.l., ) : 5

18. Quatre titres sont signalés dans Répertoire des publications gouvernementales $d u$ Québec 1867-1964 / André Beaulieu et autres (Québec: Imprimeur de la reine, 1968).

19. Signalé dans Brochures québécoises / Jean Hamelin et autres (Québec: Direction générale des publications gouvernementales, 1981).

20. Ibid.

21. Desjardins dirigea la bibliothèque à partir de 1921. Il écrivit au président du Conseil législatif le 23 novembre 1933 pour l'informer que sa santé ne lui permettait plus d'exercer ses fonctions. Son adjoint, Lucien Lemieux signe le rapport annuel de 1933 daté du 22 décembre. Marquis entra en fonction le 19 septembre 1934. Le poste fut donc vacant pendant au moins douze mois.

22. Annuaire statistique, $21^{\mathrm{e}}$ année, 1934: VI.

23. Marquis écrit : "Je n'ai pas voulu [...] retourner au Bureau des Statistiques, comme le Premier Ministre [...] me l'a offert à plusieurs reprises, avant d'avoir terminé mon travail, à la Bibliothèque. " (Rapport annuel daté du 21 mars 1950, p. 7). Par la suite, Maurice Duplessis s'est payé la tête du bibliothécaire lors de l'étude des crédits : "[...] le lieutenant-colonel G.-É. Marquis, grand amateur de statistiques, qui a été nommé probablement pour compter les volumes. » (Débats, 5 décembre 1952: 230).

24. G.É. Marquis 1950. Trois générations... : 64-79. Entre janvier 1945 et juin 1947, il fit la recension d'un ou deux titres chaque dimanche à $22 \mathrm{~h} 15$ (Lettre adressée à Roger Ouimet de Radio-Canada et datée du 8 juin 1946). Il offrit alors ses services à Radio-Canada.

25. Frère Magloire. 1947. Bio-bibliographie du colonel G.E. Marquis,... Québec: $47 \mathrm{p}$. La liste du Frère Magloire, mise à jour, est insérée dans Trois générations d'éducateurs (1950) de Marquis (p. 64-111) On l'a même indexée. La liste des brochures et des livres de l'auteur publiée dans Monuments commémoratifs de Québec (1958) énumérait vingt titres. Le catalogue de la Bibliothèque nationale du Québec en présentait 41 à l'automne 2009, dont des articles parus dans des journaux, celui de la bibliothèque de l'Assemblée nationale, 15 .

26. Le Terroir, vol. $\mathrm{XV}, \mathrm{n}^{0} 1$, juin 1933: 1 .

27. Les Fortifications de Québec... 1823-1923 (1923). Le Régime seigneurial au Canada (1931); L'Histoire du Canada commence-t-elle en 1760? (1940); Le Régiment de Lévis (1952). statut et à la qualité de la langue ${ }^{28}$. Il s'est engagé dans la communauté : Association des fonctionnaires ${ }^{29}$, comité de citoyens pour les sports d'hiver ${ }^{30}$, Société Saint-JeanBaptiste, Ligue des propriétaires, etc. ${ }^{31}$ Il présida la Société des arts, sciences et lettres de Québec en 1920, le Club canadien en $1928^{32}$, l'Institut militaire en $1929^{33}$. Il fut membre du grand conseil de l'Association canadienne des bibliothécaires en $1946^{34}$.

Les activités variées de Marquis lui ont mérité distinctions et médailles: commandeur de l'ordre du mérite scolaire (15 mai 1933) ${ }^{35}$, officier de l'Ordre français de l'Union latine ${ }^{36}$. Le Conseil de la défense nationale lui conféra le grade de colonel à la fin de son mandat (Marquis 1950, 62).

Le passage de Marquis à la Bibliothèque de la Législature, devenue la Bibliothèque de l'Assemblée nationale en 1982, fut marqué par plusieurs réalisations et par un certain nombre d'échecs. Il annonça ses intentions dans un mémoire ${ }^{37}$ daté du 13 décembre 1937, abordant successivement la classification, le luminaire ${ }^{38}$, le rapatriement et l'aménagement du sous-sol, la salle de lecture, le service des renseignements, le personnel, la loi et le règlement de la bibliothèque.

De 1936 à 1952, Marquis a assigné des secteurs de spécialisation à ses collaborateurs ${ }^{39}$. Il a réussi à boucler, en 1936, la reclassification des volumes entreprise en 1931, suspendue en 1933 et reprise en $1934^{40}$. Il a ré-ouvert la porte aux abonnés du public en 1936 (la

28. Machines à écrire sans accents (6 février 1935; 1.6); accents sur les lettres majuscules (1945); "vous nous arrivez avec une bonne douzaine de mots anglais" (19 février 1945); "le président aurait pu [...] préparer un questionnaire en français» (à Séraphin Marion, 18 nov. 1938; 2,1); suggestions de raisons sociales française adressées à dame Grenier (lettre du 15 avril 1937; dossier 1.11)

29. Un note conservée dans ses archives, probablement de 1943, contient l'objectif de doubler le nombre d'adhérents de l'Association des employés civils, calque ayant cours à l'époque (Dossier 3.3). Une autre note porte le commentaire " $100 \%$ de ses membres font partie de l'Association [...]" (Ibid.).

30. Acceptation d'une invitation de J.A. McManamy datée du 6 octobre 1936 (Archives Marquis, Dossier 2,1)

31. On trouve une longue énumération dans Les Biographies françaises d'Amérique.

32. Il en avait été vice-président en 1925 (Page de titre de Nos bibliothèques publiques).

33. Le Fait français au Canada..., p. 315

34. Il accepte le poste le 27 mai 1946 (Archives Marquis, dossier 3.11). Marquis se rend compte que la C.L.A. est neutre et il lance l'idée qu'il devrait y avoir une section catholique.

35. Le Terroir, vol. XV, $\mathrm{n}^{0} 1$, juin 1933, p.11: "[...] le Surintendant de l'Instruction publique lui décerne le diplôme de Mérite scolaire, troisième degré [...] le plus élevé [...] sur recommandation du Comité catholique du Conseil de l'Instruction publique».

36. Lettre adressée à Marquis et datée du 9 juillet 1938 Le 8 septembre, Ulric L. Gingras l'informa qu'on lui expédiait la médaille et lui demandait un chèque de 155 francs (Dossier 2.1).

37. Mémoire de cinq feuillets adressé à l'Orateur de l'Assemblée législative.

38. «[...] depuis 1915, les lecteurs se plaignent [...] les lustres ne donnent pas la moitié de la lumière suffisante [...] " (Rapport annuel daté de février 1937, p. 5).

39. Histoire, droit, canadiana, littérature (Rapport annuel daté de février 1937, p. 2).

40. Ibid. Le prédécesseur de Marquis avait fait part de la mise en branle du programme dans son rapport daté du 3 novembre 1931 et écrit qu'il serait fini "sous le plus court délai ». Marquis, trois mois après avoir pris la direction, annonce que l'entreprise sera complétée dans un an tout au plus (Rapport annuel daté du 31 décembre 1934, p. 5). Mais les fichiers 
bibliothèque leur était fermée depuis cinq ans) et il était très fier du nombre de lecteurs extérieurs et de leurs emprunts ${ }^{41}$. Il rappelait sans cesse à ses supérieurs que l'on devait recruter des employés compétents ${ }^{42}$, se plaisait à noter que des employés étaient diplômés en bibliothéconomie ${ }^{43}$ et favorisait leur participation à des cours de bibliothéconomie ${ }^{44}$. Il confia à deux dames le service aux lectrices en $1942^{45}$. L'éclairage fut améliorée ${ }^{46}$. Il entrevit l'utilité et le bien-fondé des microcartes ${ }^{47}$ et des microfilms de journaux ${ }^{48}$. Sous son règne, le comité mixte des deux chambres, duquel relevait la bibliothèque, se réunit pour la première fois en $40 \mathrm{ans}^{49}$ et les députés acceptèrent un programme de modernisa$\operatorname{tion}^{50}$ : ajustement de l'horaire de travail, aménagement du sous-sol ${ }^{51}$, modification en 1945 de la loi sur la Bibliothèque, laquelle fixait jusque-là le nombre d'employés à dix ${ }^{52}$. Le conservateur était aussi intéressé

(sujets, titres, auteurs) seront à la disposition du lectorat en 1949 ou 1950 (Rapport... daté du 21 mars 1950, p. 1).

41. Rapport annuel daté du $1^{\text {er }}$ avril 1943, p. 2; Ibid., daté du 15 avril 1947, p. 2 , etc. Au cours des douze mois de 1948, 3 ooo abonnés étaient inscrits qui ont emprunté 35000 volumes (Ibid., 22 février 1949, p. 3).

42. Il écrit au premier ministre le ${ }^{\text {er }}$ octobre 1935: «Je vous prie de bien vouloir nommer des hommes compétents [...] Il faut, au moins quelqu'un qui ait de la lecture, et même, beaucoup de lecture; qui connaisse les auteurs anciens et modernes et, de plus, qui ait des notions sur la Bibliothèque».

"Il existe aujourd'hui des écoles de bibliothécaires [...] Je pourrais recommander des candidats compétents [...]».

43. "Plusieurs employés permanents possèdent leur certificat de qualification en bibliothéconomie, de l'école des Bibliothécaires de Québec. D'autres suivent des cours chaque année » note-t-il dans son avant-dernier rapport (daté du 21 mars 1950, p. 1).

44. Réponse au Bureau fédéral de la statistique pour 1937 : deux employés ont étudié en bibliotechnie (sic). Idem en 1939 et cinq en 1944 (dossier 2.10). Marquis note dans le rapport annuel daté du 7 mars 1938 que deux chefs de service d'expérience sont allés suivre un cours à l'École des bibliothécaires de Montréal durant l'été 1937 et qu'ils continueront à l'été 1938.

45. Rapport annuel daté du $1^{\mathrm{er}}$ avril 1943, p. 2. Marquis avait proposé l'engagement de deux dames pour servir les lectrices en 1940 (Rapport daté du 28 mars 1940, p. 4). Il avait demandé la permission d'engager " une couple de dames, pour servir la clientèle féminine, attendu quiau moins les trois quarts des emprunteurs de livres sont des jeunes filles ou des dames » (Pelletier 1996, 10).

46. Extrait de l'avant-dernier rapport annuel de Marquis, celui daté du 27 février 1951 (p. 2) : « Notre système de luminaire est complet et permet [...] de lire sans éprouver de difficultés, le jour et le soir. "

47. Marquis saute si vite sur la nouvelle technique qu'il utilise les expressions "micro-card" et "micro library reader" (Rapport annuel daté du 21 mars 1950, p. 5). Dans le rapport daté du 22 février 1949, il qualifie les « micro-films » d'invention nouvelle qui apporteront économie d'espace et d'argent.

48. Coût de la reliure et espace nécessaire (parfois une verge cube par journal en comparaison de six pouces cubes pour une bobine) justifient le microfilmage (Rapport annuel daté du 19 janvier 1952, p. 2). Marquis aborde aussi le sujet dans le rapport daté du 3 mars 1948, p. 3.

49. On trouve le rapport du comité dans les Journaux de l'Assemblée, 30 mai 1940, p. 180. Le conservateur n'y avait pas été invité et, sept ans plus tard il semblait en avoir gardé de l'amertume (Rapport annuel daté du 15 avril 1947, p. 6).

50. Marquis consacre une page à la loi et aux règlements de la Bibliothèque dans son rapport daté du 28 mars 1940 (p. 7).

51. Deux salles sont aménagées au sous-sol et mises à disposition de la bibliothèque en 1942 (Rapport annuel daté du $\mathrm{r}^{\mathrm{er}}$ avril 1943, p. 6).

52. La commission de l'économie interne de l'Assemblée a adopté une résolution en ce sens le 13 mars 1945 (Archives de l'Assemblée nationale, ANQ-CDSA 502865). Référence de la nouvelle loi : L.Q., 1945, ch. 14. aux politiques ${ }^{53}$ et aux pratiques d'acquisition ${ }^{54}$ et il était particulièrement préoccupé par l'achat des Canadiana absents des rayons ${ }^{55}$. Il avait formé un comité qui se réunissait une fois par mois et qui déterminait les achats à faire ${ }^{56}$.

En dépit de ses succès, Marquis avala bien des couleuvres au cours de sa carrière de bibliothécaire. D'abord, certains collaborateurs le court-circuitaient ${ }^{57}$ régulièrement (il est vrai qu'il faisait de même à l'encontre de ses supérieurs) ${ }^{58}$ et on ne respectait pas la discipline qu'il s'efforçait d'implanter ${ }^{59}$. Il dut composer avec le système de recrutement pratiqué à l'époque ${ }^{60}$ et fut souvent obligé d'accepter des employés qu'il ne voulait pas nécessairement à la bibliothèque ${ }^{61}$, qu'on appelait parfois des "pains bénits»: ils recevaient

53. Marquis aborde régulièrement le sujet dans ses rapports annuels. Le 27 février 1951 (p. 3), il note la diminution du nombre de romans achetés et il écrit: "De plus en plus notre attention se porte vers les ouvrages que doit contenir une bibliothèque de la Législature, c'est-à-dire des livres de droit, de législation, de documentation politique. "

54. Dans le rapport annuel daté du $\mathrm{I}^{\mathrm{er}}$ avril 1943 , il aborde le sujet à plusieurs reprises : nombre de volumes achetés chaque année depuis 1936, livres achetés par classe en 1942, dons notables, abandon d'abonnements dans les années 1930, achat de livres d'occasion et de brochures, etc.

55. Il revient régulièrement sur le thème dans son rapport annuel. Un exemple dans celui daté du 28 mars 1940, p. 6.

56. Rapport annuel daté du 21 mars 1950, p. 8.

57. Extrait d'une note datée du 18 mars 1938 et adressée à Paul Sauvé : «[...] certains employés [...] acceptent des travaux, les exécutent et les livrent hors ma connaissance. Même, certaines correspondances officielles [...] me sont totalement inconnues [...] Je tiendrais à savoir si tous les employés sont tenus de se rapporter [... [ à l'autorité du conservateur, ou s'il est permis, à quelques-uns, de se tailler un fief indépendant du seigneur dans la seigneurie?» (Point 6). Douze mois plus tard, il se plaignait de ne pas connaitre la nature du travail accompli par deux fonctionnaires détachés auprès de la bibliothèque (Rapport annuel daté du 15 mars 1939, p. 3).

58. Ses archives contiennent des lettres et un certain nombre de mémoires adressés directement aux premiers ministres: un mémoire daté du $1^{\mathrm{er}}$ octobre 1935; correspondance du 30 novembre et du 5 novembre 1939, autre mémoire transmis au premier ministre le 14 juillet 1941. Et Marquis n'ignorait pas que ses patrons étaient les présidents - on les appelait " orateurs " à l'époque - du Conseil et de l'Assemblée, car le premier ministre le lui avait précisé le 27 septembre 1934 : «[...] vous relevez des orateurs-conjoints " (Dossier 1.1).

59. Des employés s'absentent sans autorisation (Rapport annuel de 1945, p. 2). Le 15 juin 1945, il écrit à l'acheteur de l'Assemblée: "[...] je vous prie de bien vouloir communiquer directement avec moi, lorsqu'il s'agira d'une question d'administration, quelle qu'elle soit. " (Dossier 3.8). Au moment où on modifie les heures d'ouverture, certains ne se soumettent pas (Lettre adressée à A. Godbout datée du 14 juillet 1941).

6o. Il écrit à une personne qui cherchait un poste: «Les nominations [...] au gouvernement de Québec relèvent de la politique [...] il faut commencer par voir son député [...] après il faut voir le ministre [...] la Bibliothèque est sous le contrôle de l'Orateur [...] je me contente d'accepter et de diriger [...] les employés que l'on dirige vers la Bibliothèque [...] " (Lettre datée du 11 mai 1950 et adressée à Irène Paquet; Dossier 5.3). Autre correspondance: "[...] je vous avoue que mes pouvoirs sont plus que limités. Les positions permanentes comme les temporaires s'obtiennent par voie de diplomatie politique. » (Lettre du 6 avril 1949 adressée à Guy Trottier; dossier 4.9).

61. En 1936, le nouveau gouvernement lui fit accepter la mise sur pied d'un service de renseignements et un titulaire (Rapport annuel daté de février 1937, p. 1). Trente mois plus tard, les Libéraux revenus au pouvoir, il commença à se plaindre du titulaire et de l'inutilité du service et classait le titulaire (et trois autres collègues) dans la liste des "disponibles " (Lettre adressée à A. Godbout, 5 décembre 1939). Au début de 1940, il identifie les employés détachés auprès de la Bibliothèque : René Garneau, Philippe Laferrière, Jean-Charles Bonenfant, Jean-Marie Bourbeau et Albert Rioux (Ibid., 20 mars 1940, p. 3). Il écrit plus tard au président de l'Assemblée: "Vous m'avez déjà soumis que la Bibliothèque était le seul endroit où l'on peut, à un moment donné, diriger un employé dont on ne savait que faire ailleurs. " (Ibid., 22 février 1949, p. 7). 


\section{Il eut maille à partir avec des hauts fonctionnaires portés à intervenir dans le domaine qu'il considérait comme le sien ou à conserver par-devers eux pendant des mois les livres empruntés.}

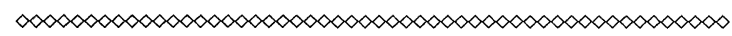

en effet de gros salaires pour faire des travaux personnels ${ }^{62}$. Ses relations avec les autorités politiques n'étaient pas à son goût, ce qui explique son langage acéré avec les présidents de l'Assemblée ${ }^{63}$ et le premier ministre ${ }^{64}$. Les hommes politiques se sont montrés fort réservés à son égard ${ }^{65}$. Marquis sollicitait beaucoup plus de rencontres qu'il n'en obtenait; on négligeait ses demandes d'ameublement ${ }^{66}$ et beaucoup de modifications matérielles proposées passaient aux profits et pertes ${ }^{67}$. Un jour, face à une rallonge budgétaire refusée pour des travaux de reliure reportés pendant les années de guerre, il interpella les autorités : "Pourquoi faut-il que la Bibliothèque... soit toujours considérée comme un enfant adoptif dont se désintéresse un père putatif ? "68. L'information administrative ne lui parvenait pas toujours ${ }^{69}$. Les membres du comité de la bibliothèque n'ont pas dû apprécier le jugement qu'il portait

62. Rapport annuel daté du 15 avril 1947, p. 4.

63. Quatre exemples: "je fais ces observations [...] pour attirer votre attention sur l'efficacité du service [...] laquelle efficacité est handicapée par le manque d'attention de ceux qui ont charge." (Lettre à Taché, 21 mars 1946: 3); «les deux Orateurs ne devraient pas être chargés de la direction de la Bibliothèque [...] puisque pendant la session ils n'ont pas le temps de s'en occuper, et qu'après ils ont encore bien d'autres chats à fouetter [...] 》 (Rapport annuel daté du 21 mars 1946, p. 3); "Vousmême, Monsieur l'Orateur, et les autres membres de la Législature, n'êtes-vous pas intéressés à savoir ce que contient votre Bibliothèque? " (Ibid., 22 février 1949, p. 2); " [...] ce ne sont pas les besoins et l'intérêt de la Bibliothèque qui sont pris en considération (par le comité de régie interne), mais plutôt les exigences du patronage. » (Ibid., p. 6).

64. Extrait d'une lettre adressée à Adélard Godbout et datée du 14 juillet 1941 : « Je savais que la Bibliothèque avait mauvaise réputation, avant que j'y entre, au point de vue discipline, travail et ordre [...] Je comprends que je dois accepter sans maugréer tous ceux qui sont dirigés vers la Bibliothèque, qu'ils soient utiles ou non [...]".

65. Lors de l'étude des crédits de l'Assemblée en février 1951, le premier ministre et le chef de l'opposition n'ont que des bons mots pour le successeur du conservateur. Marquis, de son côté, pourtant près de sa prise de retraite, est totalement ignoré (Débats de l'Assemblée législative, 13 février 1951, p. 232). Quelques mois plus tard, le premier ministre annonce que l'État accorde une augmentation de salaire à l'adjoint "appelé à remplacer assez rapidement le directeur [...]" alors qu'il n'accorde rien à Marquis et qu'il va jusqu'à dire : "[...] l'homme le plus compétent qui soit à la Bibliothèque est $M$. Jean-Charles Bonenfant.» (Débats, 5 décembre 1951, p. 230). Dans les mêmes circonstances, en 1953, on encense le nouveau directeur et on néglige le prédécesseur (Ibid., 23 janvier 1953, p. 432).

66. Cas des fichiers dont la bibliothèque avait besoin vers 1946. Marquis écrit en 1948 : "Je me suis usé la langue à demander ces meubles-là, mais rien ne vient. " (Rapport annuel daté du 22 février 1949, p. 6).

67. "[...] j’ai adressé maintes suppliques [...] au Département des Travaux publics, mais jusqu'à présent, comme sœur Anne, je ne vois rien venir." (Rapport annuel daté du 21 mars 1946, p. 3). Un an plus tard, il écrit : «On dirait que la Bibliothèque c'est l'endroit le plus négligé du Parlement. " (Rapport... daté du 15 avril 1947, p. 5).

68. Rapport annuel daté du 22 février 1949, p. 5.

69. En 1947, il se plaint de ne plus recevoir les arrêtés en conseil faisant état des nominations faites à la Bibliothèque et des traitements accordés ni sur leur proposition d'installer deux comptoirs ${ }^{70}$ pour limiter l'accès aux rayons et le voir favoriser la présence d'un policier de la Sûreté du Québec ${ }^{71}$. L'État mit fin à la publication du catalogue imprimé ${ }^{72}$ et des rapports annuels ${ }^{73}$ de la bibliothèque en $1936^{74}$ et Marquis eut beau en réclamer la reprise ${ }^{75}$, rien n'y fit ${ }^{76}$. Il eut maille à partir avec des hauts fonctionnaires portés à intervenir dans le domaine qu'il considérait comme le sien ou à conserver par-devers eux pendant des mois les livres empruntés ${ }^{77}$. Pendant qu'il intervenait en faveur de ses collaborateurs et suggérait des augmentations de salaire ${ }^{78}$, le sien, fixé à $4000 \$$ en $1934^{79}$, était toujours le même au moment de sa retraite ${ }^{80}$. Celui de son pré-

les rapports de la comptabilité de l'Assemblée (Rapport annuel daté du 15 avril 1947, p. 4).

70. Réalisés en 1940 (Rapport du ministère des Travaux publics, 1940/41, p. 29).

71. Rapport annuel daté du 28 février 1941, p. 1-2.

72. Le catalogue mis à la disposition du public avait été publié en 1905 et il était complété chaque année par un supplément annexé au rapport annuel. À partir de 1936 et jusqu'en 1941, on réalisa des listes annuelles ronéotypées des volumes achetés.

73. Le dernier rapport imprimé du conservateur couvre la période de janvier à décembre 1935 ( $23^{\mathrm{e}}$ volume). Le premier de la série fut publié en 1914. Précédemment, les rapports étaient intégrés aux procès-verbaux de l'Assemblée et du Conseil législatif. La pratique du dépôt du rapport devant les parlementaires a été abandonnée à partir des années Marquis.

74. Impression des rapports pour l'ensemble de la Fonction publique abolie, règle générale, par la "Loi concernant l'impression des rapports... "., L.Q., 1936, ch. 6.

75. En 1945, il propose la publication d'un supplément décennal 19361945 (Rapport annuel daté du 12 mars 1945, p. 2). Il revient à la charge dans une lettre adressée à Alexandre Taché datée du 5 décembre 1945. Au printemps 1947, il reconnaît n'avoir toujours pas eu d'autorisation (Rapport annuel daté du 15 avril 1947, p. 5).

76. En avril 1937, Marquis écrit deux fois au président Sauvé à ce sujet (Lettres du 2 et du 16 avril (Dossier 1.1). Il note dans son rapport annuel daté 21 mars 1946 (p. 2) qu’on ne lui a pas accordé la permission de publier un rapport décennal. En mars 1943, un rapport septennal était en préparation qu'il avait l'intention de publier après mai 1944 (Lettre datée du 23 mars 1943 adressée à A.L. Shaw; dossier 3.4).

77. Il avait dit de Jean Bruchési qu'il avait des «[...] allures d'un écrivain qui croit posséder la science infuse " (Rendez à César...; Québec, 1940, p. 4). Marquis réclamait, exigeait, devrait-on dire, des volumes achetés par le Secrétariat et destinés aux écoles. Un jour, Bruchési le semonça: "À défaut de politesse, vous pourriez tout au moins avoir un peu de jugement." (Lettre adressée à Marquis et datée du 20 janvier 1943; dossier 3,3; incident raconté par J.G. Pelletier 1996:12). Le président Laferté note, dans son journal, à la date du 18 janvier 1940: «Téléphone de Marquis [...] me dit que Jean Bruchési avait voulu avoir le contrôle de la bibliothèque [...] ajoute qu'il ne veut pas passer sous le contrôle de Jean Bruchési [...] " (Derrière le trône; Mémoires d'un parlementaire,,; Québec: Septentrion, 1998, p. 146). De plus, on peut supposer que d'excollaborateurs d'élus, détachés auprès de la Bibliothèque, n’appréciaient pas qu'on les désigne comme des "êtres inutiles " (Lettre datée du 30 novembre 1939 et adressée au premier ministre).

78. Voir lettre au premier ministre datée $d u{ }^{2 \mathrm{er}}$ octobre 1935. Marquis écrit à Paul Sauvé le 7 mai 1937 en faveur de quatre employés (Dossier 1.1). Il intervient en faveur d'une collaboratrice auprès du président du Conseil législatif le 5 février 1942 (Dossier 2.9). Le premier ministre Duplessis affirmait d'ailleurs que l'augmentation moyenne des salaires à la bibliothèque de 1944 à 1951 avait été de 355 \$ par tête (Débats de l'Assemblée législative, 13 février 1951, p. 232). Mais celui de Marquis n’a pas bougé.

79. Le décret 2234 daté du 19 septembre 1934 ne contient pas d'autre précision que la suivante : "Que M. G.-E. Marquis [...] soit nommé bibliothécaire [...] avec un traitement annuel de $\$ 4000.00$, en remplacement de $M$. Joseph Desjardins, démissionnaire. » Le traitement fixé en 1934 était celui des sous-ministres d'alors, lesquels gagneront 8000 \$ en 1948 .

80. Comme compensation sans doute, l'Assemblée acheta pour la somme de $1200 \$$ la bibliothèque personnelle de Marquis, laquelle comptait plus de 1100 volumes (Archives de l'Assemblée nationale, Fonds Bonenfant, dossier 1.2.1). 
décesseur avait pourtant augmenté de $50 \%$ entre 1921 et $1934^{81}$ et son propre adjoint gagnait $12,5 \%$ de plus que lui en $1951^{82}$. Son langage direct et son esprit militaire l'ont à coup sûr desservi.

Le conservateur utilisait un langage direct et sans détour avec les lecteurs, mais il était fort prudent à l'occasion. À un fonctionnaire qui ne remettait pas un livre, il écrit: "si votre Département a besoin de certaines publications à la longue année, il n'a qu'à les acheter. ${ }^{83}$ Il reprend l'affirmation à l'égard d'un citoyen six ans plus tard ${ }^{84}$. À un correspondant qui l'avait désigné par l'expression "gardien de...", il conseille d'adresser ses lettres au Conservateur ${ }^{85}$. Il juge ne pouvoir acheter les livres d'un auteur "plutôt dévergondé » 86 et il admet à la réserve, c'est-à-dire à l'enfer, seulement les hommes " ayant fait un cours classique complet ${ }^{87}$; il distingue trois collections distinctes : livres à l'index, livres réservés ou dangereux et livres d'usage général ${ }^{88}$. Il suit les instructions de l'Index librorum prohibitorum et il tient pour acquis que les rayons de la bibliothèque contiennent à la fois "des ceuvres de vie et des ouvres de mort "89. Il méprise certaines catégories de personnes et ne souffre pas de cacher ses sentiments ${ }^{90}$. On doit choisir ses sujets avec lui ${ }^{91}$ note un abonné. Et une notice biographique, datée de 1953, précise: «Homme d'étude que les futilités exaspèrent. ${ }^{92}$

Marquis se fit le promoteur de nombreux projets au cours de son passage à la bibliothèque des parlementaires : dépôt légal payé9 ${ }^{33}$, dépôt automatique des publi-

81. 2000 sen $1921 / 22$ et 3000 s en $1933 / 34$.

82. Cette année-là, Marquis toucha $4000 \$$ et Bonenfant, $4500 \$$. Il aborde le sujet dans le dernier rapport annuel qu'il signe et qui est daté du 19 janvier 1952 : il dit que son adjoint mérite l'augmentation mais note que son propre salaire est "resté ce qu'il était il y a dix-sept ans». (p. 1-2). Pelletier (1996) consacre un paragraphe à ce fait. Selon les comptes publics, le salaire de J.C. Bonenfant est passé de $4645 \$(1952 / 53)$ à 18400 $\$(1968 / 69)$.

83. Lettre adressée à Henri Kieffer datée du 23 avril 1940. Et Marquis termine en disant: "[...] si tous nos emprunteurs faisaient comme vous, il n'y aurait plus bientôt, dans la Bibliothèque, que les colonnes et les rayons." En 1937, il avait écrit dans le même sens au secrétaire particulier d'un ministre qui voulait garder par-devers lui un volume dont il avait encore besoin (Lettre datée du 24 décembre 1937 adressée à Wheeler Dupont).

84. "Si vous avez besoin d'un livre à l'année, vous n'avez qu'à l'acheter." (Lettre datée du 10 septembre et adressée à Charles Bédard).

85. Lettre adressée au Frère Jean-Lucien (Asbestos) et datée du 23 août 1949.

86. Témoignage sur la Bibliothèque (Québec : la Bibliothèque, 2002) : 68 .

87. Ibid., p. 69. Fernand Dumont raconte dans ses mémoires une entrevue avec Marquis (Récit d'une émigration; mémoires; Montréal : Éditions du Boréal, 1997, p. 48-49). Le passage se trouve dans Témoignage sur la Bibliothèque, 47.

88. Rapport annuel daté de février 1937, p. 8. La même typologie est reprise dans le rapport daté du 15 mars 1939, p. 10.

89. Rapport annuel daté du 15 mars 1939, p. 10-11.

90. Il écrit à un correspondant de Beauport (14 novembre 1944): «[..] pourquoi chercher la compagnie des imbéciles ou des égoistes, quand il y a tant de variétés dans les livres?" (Dossier 3.6).

91. "Monsieur le colonel [...] venait causer avec nous. Comme il s'était plaint "d'Au pied de la Pente douce" (ce dieu n'est-il pas le sien?) parce que Lemelin avait parlé de leur dieu, mieux valait choisir nos sujets." (Raymond Deraspe, dans Témoignages sur la Bibliothèque, 40).

92. Le Fait français au Canada (Montréal: Société nouvelle de publicité, 1953) : 315 .

93. "La Législature ne devrait-elle pas décréter que chaque éditeur enverra, contre remboursement, deux exemplaires de toute brochure ou de tout livre qu'il mettra à jour?» (Rapport annuel, 1935; Québec, 1936, p. 6).
Il suit les instructions de l'Index librorum prohibitorum et il tient pour acquis que les rayons de la bibliothèque contiennent à la fois " des œuvres de vie et des oeuvres de mort ».

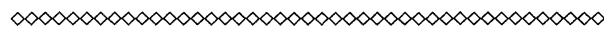

cations de l'État auprès de la bibliothèque $\mathrm{e}^{94}$, enregistrement de tous les livres et publication d'un bulletin de bibliographie ${ }^{95}$, lequel faciliterait le travail de sélection des titres à acquérir ${ }^{96}$, indexation de journaux avant que la pratique devienne courante ${ }^{97}$, établissement d'un service postal de prêts ${ }^{98}$, création d'une section scolaire à la bibliothèque ${ }^{99}$, formation d'un comité chargé d'encourager la mise sur pied de bibliothèques dans les centres populeux ${ }^{100}$, aménagement du soubassement ${ }^{101}$ et de bureaux superposés aux extrémités de la bibliothèque $^{102}$. Marquis tenait à la publication d'un catalogue imprimé puisque le fichier «ne saurait être à la portée de ceux qui n'habitent pas Québec » ${ }^{103}$. Il recommanda en 1936 la célébration du centième anniversaire de naissance de Pamphile LeMay ${ }^{104}$ et, en 1943, celle

Trois ans plus tard, Marquis écrit : «Il serait désirable [...] que les éditeurs déposassent, à la Bibliothèque [...], au moins deux exemplaires de leurs ouvrages, contre remboursement [...]» (Rapport annuel daté du 15 mars 1939, p. 8). En 1945, le Maître Imprimeur reprend l'idée en la modifiant quelque peu (Voir Jean-Pierre Chalifoux, L'Édition au Québec 1940-1950; Université de Montréal, École de bibliothéconomie; Mémoire; juin 1973, p. 38).

94. Quatrième point d'une lettre adressée à Paul Sauvé le 18 mars 1938: "M'autorisez-vous à adresser une lettre officielle à tous les ministres, pour leur demander qu'une distribution soit faite automatiquement et régulièrement à la suite de l'impression de toute publication officielle? ». Pelletier (1996 : 13) écrit, en s'appuyant sur une lettre de Marquis datée du 14 septembre 1940 et adressée à Hector Laferté «[...] non sans difficultés, Marquis obtint que les ministères lui fissent parvenir leurs documents. "

95. Rapport annuel de 1936 daté de février 1937, p. 2. Marquis revient sur le sujet dans son rapport daté du 7 mars 1938 : "J'ai déjà suggéré [...] l'enregistrement, au siège du Gouvernement [...], de tous les nouveaux livres [...] Une autre lacune à combler [...] serait la création d'un Bulletin bibliographique.» $(\mathrm{p}, 5)$.

96. Rapport annuel daté du 15 mars 1939, p. 8.

97. Rapport annuel de $1936 \ldots$, p. 2.

98. Lettre datée du 4 janvier 1943 adressée à M.A. Breton de Lac-Mégantic (dossier 3.3).

99. Rapport annuel daté du $1^{\mathrm{er}}$ mai 1936, p. 5; Ibid., février 1937, p. 3.

100. Lettre datée du 30 janvier 1950 et adressée au trésorier de l'A.C.B.F., l'abbé Irenée Sauvé.

101. À son arrivée à la bibliothèque, Marquis proposa que les ministères libèrent l'espace qu'ils y occupaient. Simultanément, il fit part de la pauvre qualité de la ventilation et de l'humidification (Rapport annuel daté du 28 mars 1940, p. 2). Il revient sur le sujet en 1949 (Ibid., 28 février 1949, p. 2). Les travaux de réfection furent réalisés après sont départ.

102. En 1948, Marquis proposa qu'on fasse des bureaux supplémentaires à l'entresol (Le Soleil, 6 avril 1948). Il avait abordé le sujet dans le rapport annuel daté du 28 mars 1940 (p. 2) et dans celui daté du 3 mars 1948 (p. 4). Dans ce dernier rapport, il lança l'idée d'en faire des «chambres d'étude pour les professeurs et les étudiants ». Il fit de même dans celui daté du 21 mars 1950 (p. 5). Par la suite, J.C. Bonenfant, dans les années 1960, et un comité interne, en 1986, avaient fait leur la suggestion. Les bureaux furent aménagés en 2000! (Bulletin de la Bibliothèque..., vol. $34, \mathrm{n}^{\mathrm{s}} 3-4$, novembre 2005, p. 22).

103. Rapport annuel daté du 13 mars 1945, p. 2.

104. Sixième bibliothécaire des parlementaires depuis 1829. Marquis suggéra un monument destiné à une place publique (Rapport annuel daté du $1^{\mathrm{er}}$ mai $\left.1936, \mathrm{p} .7-8\right)$. Un an après, il parlait «d'une simple colonne de 
Sa personnalité, son conservatisme, son langage direct, son manque d'entregent, sa difficulté à accepter les compromis, son obsession de la hiérarchie et son esprit militaire l'ont cependant desservi.

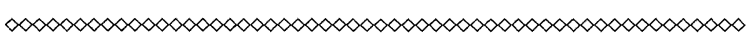

de la première édition de l'Histoire de François-Xavier Garneau (1845) ${ }^{105}$.

Les principes à la base de la carrière documentaire de Marquis sont de l'époque mais il leur apporta une touche personnelle. Il était en faveur du recrutement de spécialistes et, peu de temps après sa nomination il défendit son point de vue ${ }^{106}$. Il considérait que la profession est «le paradis sur la terre" pour quiconque aime les livres ${ }^{107}$. Il ne demandait pas mieux que de rendre service ${ }^{108}$ et croyait à l'utilité des bibliothèques de lecture publique dont il entrevoyait le rôle social $^{109}$; il en encourageait la mise sur pied ${ }^{110}$ et acceptait de leur acheminer des dons ${ }^{111}$. Malgré tout, il assimilait les bibliothèques à des arsenaux ${ }^{112}$, à des dépôts d'œuvres «de vie " mais aussi d'œuvres "de mort» ${ }^{113}$. Aux étudiants qui voulaient emprunter des livres, il demandait la signature des autorités pédagogiques ${ }^{114}$.

granit surmontée du buste [...]" (Ibid., février 1937, p. 6), œuvre qui ressemble à celle signée Henri Hébert en 1929 et remise à la bibliothèque deux ans plus tard (Maurice Pellerin, Pamphile LeMay, bibliothécaire...; Québec : la Bibliothèque, 1986, p. 21-22). Marquis ne parle pas du projet dans son rapport daté du 7 mars 1938 ni dans celui du 15 mars 1939.

105. Rapport annuel daté du $1^{\mathrm{er}}$ avril 1943, p. 13.

106. Dans un mémoire, daté du $1^{\mathrm{er}}$ octobre 1935, adressé au premier ministre

Taschereau, il écrit à propos du personnel : "Il faut, au moins quelqu'un qui ait de la lecture [...] qui ait des notions sur la Bibliothèque. " et, plus loin, "Il existe aujourd'hui des écoles de bibliothécaires [...] comme il existe une école des Hautes études commerciales [...]».

107. Lettre adressée à Fernande Villemaire datée du 20 février 1945 (Archives Marquis, dossier 3.7). Précédemment, il avait écrit à ses supérieurs: "J'aime le poste que j'occupe et je n'en connais pas où l'atmosphère soit plus favorable à celui qui aime les livres et le commerce intellectuel avec les écrivains. " (Rapport annuel daté de février 1937, p. 8).

108. "C'est notre seule raison d'être" écrit-il à un correspondant de l'Université Laval (lettre adressée à Alexandre Marcotte datée du 31 décembre 1945 (Archives Marquis, dossier 3.10).

109. "Quelqu'un a dit que bâtir une école c'est fermer une prison. Ne pourrait-on pas ajouter... qu'organiser une bibliothèque paroissiale c'est fermer une buvette ou tout autre endroit néfaste?" (Lettre adressée au R.P. Lamontagne, datée du 7 janvier 1944; Archives Marquis, dossier 3,3); "Sans bibliothèque, les habitants d'un pays n'ont pas tous les organismes nécessaires à l'expression d'une civilisation moderne " (Rapport annuel daté du 21 mars 1950, p. 9).

110. "Je vous félicite de l'idée que vous avez de fonder une bibliothèque paroissiale ", écrit-il à l'abbé Arthur Gingras (Sainte-Justine) le 21 février 1946 (Archives Marquis, dossier 3.10).

111. Ibid. Autre don fait au R.P. Lamontagne pour la bibliothèque paroissiale de Saint-Cœur-de-Marie (Lettre datée du 7 janvier 1944; Archives Marquis, dossier 3,3).

112. "Toutes les bibliothèques générales ou techniques sont appelées à rendre des services [...] C'est un arsenal qui contient des armes offensives et défensives, mais [...] il faut connaitre le travail que chaque pièce peut accomplir et de plus la technique de chacune des armes de combat." (Lettre à Edgar Rochette datée du 22 novembre 1943; Archives Marquis, dossier 3.5).

113. Rapport annuel daté du 28 mars 1940, p. 10.

114. Et il affirmait : "l'éducation des jeunes gens ne doit pas être influencée par des lectures qui ne cadrent pas avec les études qu'ils poursuivent. » (Lettre
Tout militaire qu'il fut, il savait que les bibliothèques dépendent pour beaucoup des désidératas de la population: en 1946, lors d'une conférence, il encouragea les pressions d'un regroupement de fonctionnaires en matière de locaux et d'éclairage, de collections et de catalogues $^{115}$. Il semblait regretter que les livres soient à la portée des lecteurs et il alla même jusqu'à suggérer des rayons fermés et blindés ${ }^{116}$, formule qui aurait empêché le sabotage du classement ${ }^{117}$.

L'histoire a retenu surtout deux faits de la carrière de Marquis. D’abord, le chassé-croisé auquel sa nomination a donné lieu (Pelletier 1996, 9). Deux candidats étaient en lice pour prendre la succession de Joseph Desjardins, malade depuis douze ou quinze mois: Paul Gouin et Jean-Charles Harvey. Ce dernier avait été rédacteur en chef du Soleil et il avait publié en 1934 un roman, Les Demis-civilisés, que les autorités ecclésiastiques de Québec avaient mis à l'index. Aussi s'opposa-t-on à sa nomination ${ }^{118}$. Quant à Gouin, il déclara forfait et Marquis devint la bouée de sauvetage du gouvernement Taschereau. Selon Jean-Louis Gagnon, il avait la tête de l'emploi, il se voyait comme un chef, portait beau et ne sortait jamais sans sa canne même s'il n'était pas un foudre de guerre ni un intellectuel ${ }^{119}$. On le muta à la bibliothèque et on nomma Harvey, avec un salaire équivalent à celui qu'il recevait au Soleil ${ }^{120}$, à la tête du Bureau de la statistique ${ }^{121}$, « un compromis [...] qui frisait le ridicule » a-t-on écrit ${ }^{122}$. En dépit des nombreuses réalisations et de l'éclectisme de Marquis, de son amour des livres ${ }^{123}$, de ses nombreux centres d'intérêts et des statistiques qu'il avait établies sur les bibliothèques ${ }^{124}$, on retint surtout qu'il ne connaissait rien au

datée du 20 septembre 1943 et adressée au R.P. Robert Bernier, c.j.e.; dossier 3.5). Il précise la pensée des supérieurs de maison d'enseignement dans le rapport annuel daté du 28 mars 1940 (p. 9).

115. La Bibliothèque de la législature; son passé, son présent, son avenir (Québec, 1946), p. 26-30.

116. Rapport annuel daté du 15 mars 1939, p. 2. Plus loin, il écrit: "L'un des grands inconvénients d'une bibliothèque ouverte, c'est que nos clients (sic)[...] vont faire leur choix eux-mêmes dans les rayons, sans attendre qu'un commis les accompagne." (p. 5). Marquis revient sur le sujet dans le rapport daté du 28 mars 1940 (p. 1 et 5).

117. Rapport annuel daté du 15 avril 1947, p. 3 .

118. L'archevêché de Québec acceptait que Harvey soit nommé à un poste de la Fonction publique, sauf au Conseil de l'instruction publique ou à la Bibliothèque du Parlement (Jean-Louis Gagnon, Les Apostasies, tome 1: Les coqs de village; Montréal : La Presse, 1985, p. 93).

119. Ibid., p. 94.

120. Ibid., p. 93 .

121. Le gouvernement de l'Union nationale le limogea en février 1937. Harvey raconte toutes les péripéties dans l'introduction qu'il a rédigée en 1966 et reproduite dans les nouvelles éditions (par exemple Montréal : Typo, 1996, 2009, p. 7-11).

122. Guildo Rousseau, dans Dictionnaire des œuvres littéraires du Québec / sous la direction de Maurice Lemire (Montréal: Fides, 1980), vol. II, p. 347 .

123. Il écrivit à Félix Desrochers, son nouvel homologue fédéral, le 26 septembre 1934, lequel l'avait félicité de sa nomination : « [...] les livres ont toujours été pour moi mes meilleurs amis. » (Dossier 1,5).

124. On a même écrit au moment de sa mutation: "Le colonel Marquis apportera comme bibliothécaire le fruit de quinze années de recherches sur les bibliothèques de la province, qu'il doit d'ailleurs recueillir en volume bientôt. " (Le Soleil, 20 septembre 1934). 
domaine et que son successeur au BSQ ne connaissait rien à la statistique ${ }^{125}$.

Un second élément notable de la carrière de Marquis est qu'il semble avoir inspiré un personnage du roman Au-delà des visages ${ }^{126}$ d'André Giroux. Les modèles possibles dans les années 1940 n'étaient pas nombreux dans la capitale et même au Québec. JeanCharles Bonenfant, collaborateur de Marquis durant une décennie, a prudemment reconnu la ressemblance entre le personnage et le bibliothécaire ${ }^{127}$, lequel apparaît sous les traits d'un réel conservateur ${ }^{128}$; Marquis a cependant fait un compte rendu ${ }^{129}$ élogieux du roman.

La présence de Marquis à la tête de la bibliothèque de la représentation nationale fut à la mesure et à la hauteur de celle de ses prédécesseurs et de ses successeurs. Sous sa direction, l'établissement a progressé et il est resté contemporain. À son arrivée, on y trouvait une collection de 162 ooo volumes et, au moment de sa retraite, presque $200000^{130}$. Pendant la même période, le nombre d'employés est passé de dix à vingt-deux permanents ${ }^{131}$, les crédits annuels de $30000 \$$ à près de $67000 \$$, les sommes réservées à l'achat des volumes et à la reliure d'un peu moins de 10000 à 15000 \$. Marquis a proposé des idées que ses successeurs ont adoptées et réalisées ${ }^{132}$ comme il avait lui-même assumé l'héritage qu'on lui avait confié en 1934. Sa présence et son engagement dans de nombreux milieux, ses multiples écrits, ses conférences et sa présence radiophonique font de

125. La scie suivante court toujours : Marquis, qui ne connaissait rien aux livres fut nommé à la bibliothèque et Harvey, qui ignorait tout de la statistique, au Bureau de la statistique. Elle apparaît dans l'introduction au roman signée par Harvey et insérée dans l'édition de 1966 (Éditions de l'Homme). Louis-Guy Lemieux la reprend dans un article intitulé « La bombe des Demi-civilisés " (Le Soleil, 12 mai 1996, p. B-3). L'archiviste de l'Assemblée nationale, à l'époque, avait écrit au journaliste: «[...] Marquis... n'était pas aussi étranger au monde du livre que l'a dit JeanCharles Harvey lors de la réédition de son roman [...] c'était même un bibliophile. Par contre, je suis d'accord [...] Harvey n'était pas à sa place à la Statistique» (Lettre de Jean-Guy Pelletier datée du 13 mai 1996).

126. Québec, les Éditions Variétés, 1948, 172 p. Un chapitre porte le titre «Le bibliothécaire » (p. 61-69).

127. "Dans Au-delà des visages, André Giroux a campé un portrait légèrement cruel d'un bibliothécaire du Parlement qui, pour des lecteurs avertis, ressemblait quelque peu à Georges-Émile Marquis. " (Livres, bibliothèques et culture québécoise; Montréal : Asted, 1977, p. 649).

128. Quelques phrases révélatrices de la pensé du bibliothécaire : "Jeune homme, ne vous moquez pas de l'Enfer. C'est un paratonnerre "; «[...] moi, j'ai un sacerdoce intellectuel à exercer ici»; "La culture, $j$ 'en suis! Mais d'abord, la morale! " (Au-delà des visages, p. 62, 66 et 68).

129. L'Action, 24 novembre 1948 , p. 12.

130. Le nombre de volumes est donné dans les rapports annuels. À la fin de 1951, $199877 ; 165$ ooo en octobre 1935.

131. En dépit de la boutade du premier ministre Duplessis le 5 décembre 1951, laquelle laisse à entendre que l'effectif aurait diminué : "Il fut un temps où il y avait à la Bibliothèque plus d'employés que de volumes. " (Débats, p. 230). Mais de fait, le nombre d'employés est passé de 26 à 17 de 1944 à 1945, du gouvernement Godbout au gouvernement Duplessis.

132. Les premiers microfilms de journaux furent achetés dans les mois qui ont suivi sa retraite. Idem d'une liseuse (Rapport annuel daté 5 février 1953, p. 2). Le dépôt légal des publications de l'État au profit de la bibliothèque de la représentation nationale fut décrété dans les années 1980 (Article 132 de la Loi sur l'Assemblée nationale). Le bulletin bibliographique apparut à la fin des années 1960 à l'initiative de la Bibliothèque nationale et de Georges Cartier. Les index de journaux évoqués dans les années 1930 devinrent réalité vers 1965 (Index du Devoir). Idem de la climatisation des locaux et des magasins évoquée au début de 1948 (Rapport annuel daté du 3 mars 1948), etc. lui un professionnel exemplaire. Sa personnalité, son conservatisme, son langage direct, son manque d'entregent, sa difficulté à accepter les compromis, son obsession de la hiérarchie et son esprit militaire l'ont cependant desservi. Il fut un "mécontemporain » et, sûrement, un électron libre, comme on l'aurait dit à la fin du $\mathrm{XX}^{\mathrm{e}}$ siècle. Si l'un des canons du rayonnement est « bien faire et le faire savoir », Marquis n'aura pas réussi à faire mousser ses réalisations. -

\section{Sources consultées}

Marquis, Georges-Émile.1925. Nos bibliothèques publiques, Québec, Imprimerie du Soleil, 16 p. (causerie donnée au Château Frontenac le 4 mai 1925 devant les membres de la section française de l'Association des auteurs canadiens).

Marquis, Georges-Émile.1934. La Bibliothèque, S.1., s.n., 15 p. (causerie donnée au congrès de l'Union des municipalités..., le 27 août 1934, à bord du Richelieu.

Marquis, Georges-Émile. 1946. La Bibliothèque de la législature, son passé, son présent, son avenir..., Québec, s.n., 31 p. (Dîner-causerie au Chalet des employés civils, le 27 février 1946).

Marquis, Georges-Émile. 1950. Trois générations d'éducateurs, Québec, s.n., : 132 p.

Magloire, Frère. 1947. Bio-bibliographie du colonel G.É. Marquis, Québec, 47 p.

Pelletier, Jean-Guy. 1996. G.É. Marquis, conservateur de la Bibliothèque; un tempérament impétueux. Bulletin de la Bibliothèque de l'Assemblée nationale, vol. 26, $\mathrm{n}^{\text {os }}$ 2-3, août : 9-13.

Pelletier, Jean-Guy. 1994. Fonds Georges-Émile Marquis. Association québécoise d'histoire politique, Bulletin, vol. $2, \mathrm{n}^{\circ} 3$, hiver : 399-403.

Poirier, Paul. 1993. Répertoire numérique simple du fonds GeorgesÉmile Marquis, Québec, Assemblée nationale, Direction de la Bibliothèque, Service des archives, $43 \mathrm{f}$.

Rochette, Christian. 2002. Georges-Émile Marquis, un conservateur réformiste à la Bibliothèque de la législature de 1934 à 1940, dans Au fil des pages et du temps, la Bibliothèque de l'Assemblée nationale deux siècles d'histoire, sous la direction de Gilles Gallichan, Bibliographie et documentation, 44, Québec, la Bibliothèque, 45-55. 